\title{
Automatic Point Landmark Matching for Regularizing Nonlinear Intensity Registration: Application to Thoracic CT Images ${ }^{\star}$
}

\author{
Martin Urschler ${ }^{1}$, Christopher Zach ${ }^{2}$, Hendrik Ditt ${ }^{3}$, and Horst Bischof ${ }^{1}$ \\ ${ }^{1}$ Institute for Computer Graphics \& Vision, Graz University of Technology, Austria \\ \{urschler, bischof\}@icg.tu-graz.ac.at \\ ${ }^{2}$ VRVis Research Centre, Graz, Austria \\ zach@vrvis . at \\ ${ }^{3}$ Siemens Medical Solutions, CTE PA, Forchheim, Germany \\ hendrik.ditt@siemens.com
}

\begin{abstract}
Nonlinear image registration is a prerequisite for a variety of medical image analysis tasks. A frequently used registration method is based on manually or automatically derived point landmarks leading to a sparse displacement field which is densified in a thin-plate spline (TPS) framework. A large problem of TPS interpolation/approximation is the requirement for evenly distributed landmark correspondences over the data set which can rarely be guaranteed by landmark matching algorithms. We propose to overcome this problem by combining the sparse correspondences with intensity-based registration in a generic nonlinear registration scheme based on the calculus of variations. Missing landmark information is compensated by a stronger intensity term, thus combining the strengths of both approaches. An explicit formulation of the generic framework is derived that constrains an intra-modality intensity data term with a regularization term from the corresponding landmarks and an anisotropic image-driven displacement regularization term. An evaluation of this algorithm is performed comparing it to an intensity- and a landmark-based method. Results on four synthetically deformed and four clinical thorax CT data sets at different breathing states are shown.
\end{abstract}

\section{Introduction}

A large number of medical image analysis applications require nonlinear (deformable) registration of data sets acquired at different points in time. Especially when dealing with soft tissue organs, like the lung or the liver during breathing, motion differences have to be compensated for further analysis steps. Surveys on nonlinear registration methods in medical imaging can be found in Maintz and Viergever [1] or Crum et al. 2]. The literature distinguishes between intensityand feature-based nonlinear registration methods. Often feature-based methods are more accurate as long as the feature extraction or segmentation steps are reliable. Due to the reduction of the problem space, feature-based methods are

\footnotetext{
* This work was funded by Siemens MED CT, Forchheim and Siemens PSE AS, Graz.
} 
also significantly faster to compute. However, inaccuracies from the often difficult feature extraction step have severe effects on the registration performance, making the intensity based methods perform better in many practical applications. Feature-based registration often uses manually or automatically derived point landmarks 3]. After matching the resulting sparse displacement field from the point correspondences is interpolated/approximated in the TPS 43 framework which crucially depends on an even distribution of point correspondences over the data set. However, this even distribution can rarely be guaranteed by automatic matching algorithms. Building upon our previous work on automatic point landmark extraction, matching and registration [5] the goal of this work is to replace the TPS displacement field estimation by intensity-based registration, thus establishing a hybrid nonlinear registration scheme. The assumption that the coupling of landmark- and intensity-based registration is more powerful than the individual steps has already been investigated by other authors. Gee et al. 6] proposed a Bayesian framework to unify these approaches. Johnson and Christensen [7] combined landmark thin-plate spline registration with intensity registration using a viscous fluid regularization in a consistent manner. In Li et al. 8] consistent intensity registration was combined with airway tree branch point matching for deformable lung registration. Fan and Chen 9] initialized an optical flow based intensity registration method with a semi-automatic airway tree and lung segmentation. Hellier and Barillot [10 proposed a unified brain registration framework combining a global intensity approach with sparse landmark constraints. Liu et al. [11] showed a combined volumetric and surface matching approach for inter-subject brain registration. Papademetris et al. [12 recently proposed a hybrid registration combining B-Spline free-form deformation intensity registration with the Robust Point Matching algorithm. Many of these hybrid schemes need a large computational effort during registration or during automated segmentation. Others require manual landmark selection or tedious semi-automatic segmentation methods for pre-processing. In this work we present a novel fully-automatic hybrid nonlinear registration framework that is both efficient and does not require pre-processing. This framework uses a flexible variational formulation 13 , where different data and regularization terms can be used and the corresponding landmarks from the automatic landmark matching [5] act as an additional regularization constraint.

\section{Landmark-Based Registration}

In [5] an automatic landmark extraction, matching and registration approach consisting of a four-stage pipeline was constructed, inspired by state of the art computer vision matching techniques [14. First, a robust and reproducible point landmark extraction step based on 3D Förstner corner detection [3] is used which produces a large number of landmark candidates. Second, for each landmark candidate a local and a global descriptor is calculated. The local descriptor is a 3D extension of the widely-used SIFT descriptor representation [15, which can be interpreted as a local gradient location and orientation histogram. To capture 
global correspondence information an approximated 3D shape context descriptor [16] is computed. For each point landmark shape context can be interpreted as a quantized distribution of relative distances to all other points in the set of landmark candidates. Given these two descriptors a cost function is formed in the third stage and a robust, conservative forward-backward matching approach is applied to find a set of sparse correspondences. Each correspondence is assigned a matching uncertainty value derived from the matching cost function. The final stage of the registration pipeline is a dense displacement field estimation in a TPS framework 4, where ideas from Rohr 3 are used to approximate the interpolation condition and to incorporate the matching cost uncertainties. A k-d tree based approximation of the TPS model is proposed to save computation time. However, the algorithm, while being very efficient in the first three stages, still needs large computational effort for the final dense displacement field estimation and shows a crucial dependence on the even distribution of matched landmarks over the input volume.

\section{Intensity-Based Registration}

The general nonlinear registration problem can be formulated as a minimization process of a cost functional that depends on a similarity function $\mathcal{S}$ and a regularization constraint $\mathcal{P}$ [13. Reference image $R$ and floating image $F$ are considered as intensity functions $R: \Omega_{R} \rightarrow \mathbb{R}$ and $F: \Omega_{F} \rightarrow \mathbb{R}$ over the domains $\Omega_{R}, \Omega_{F} \subset \mathbb{R}^{3}$. The formalized registration problem is now stated as the problem of finding a displacement field $(u, v, w)^{T}: \Omega_{R} \rightarrow \Omega_{F}$ that minimizes (for a weight $\lambda>0$ ) the continuous cost functional

$$
\mathcal{J}[u, v, w]=\iiint \mathcal{S}[u, v, w]+\lambda \mathcal{P}[u, v, w] d x d y d z .
$$

A widely used choice for a similarity measure $\mathcal{S}$ is the $L_{2}-$ norm of the intensity differences in intra-modality applications. With a regularization term $\mathcal{P}$ that minimizes the norm of the displacement field gradients in an image-driven anisotropic fashion [17] an optical flow registration scheme can be derived [910]. Minimization can be achieved by solving the Euler-Lagrange equations e.g. using a Gauss-Seidel fixed-point iteration scheme.

\section{Hybrid Landmark and Intensity Registration}

The landmark-based registration approach [5] works well as long as an evenly distributed set of correspondences can be found. To avoid this shortcoming and to properly combine the advantages of landmark- and intensity-based registration such that both approaches concurrently contribute to the optimal solution, we propose to use the sparse set of matched landmarks as an additional regularization constraint $\mathcal{Q}$ in the general nonlinear registration functional. This leads to the continuous cost functional (for weights $\lambda>0, \mu>0$ )

$$
\mathcal{J}[u, v, w]=\iiint \mathcal{S}[u, v, w]+\lambda \mathcal{P}[u, v, w]+\mu \mathcal{Q}[u, v, w] d x d y d z
$$


The $L_{2}-$ norm data term is defined as

$$
\mathcal{S}[u, v, w]=(1-W(x, y, z))(R(x, y, z)-F(x+u, y+v, z+w))^{2},
$$

where the factor $(1-W(x, y, z))$ penalizes the data term near landmark correspondences. Anisotropic image-driven regularization is given as

$$
\mathcal{P}[u, v, w]=\nabla u^{T} D(\nabla F) \nabla u+\nabla v^{T} D(\nabla F) \nabla v+\nabla w^{T} D(\nabla F) \nabla w,
$$

where $D(\nabla F)$ is the diffusion tensor that creates a dependency between the estimated displacement field and the image boundaries of the floating image such that regularization across image boundaries is penalized. Finally the landmark matching constraint is

$$
\mathcal{Q}[u, v, w]=W(x, y, z)\left\|(u, v, w)^{T}-\left(u_{f}, v_{f}, w_{f}\right)^{T}\right\|^{2} .
$$

$W(x, y, z)$ is a weighting function incorporating landmark correspondences and their matching uncertainties and $\left(u_{f}(x, y, z), v_{f}(x, y, z), w_{f}(x, y, z)\right)^{T}$ is the sparse displacement field from the automatic landmark matching step. Note that the additional landmark matching regularization term is independent of the explicit choice of data or regularization terms.

Minimization of the cost functional $\mathcal{J}$ is achieved by setting the functional derivatives of $\mathcal{J}$ equal to 0 resulting in a coupled system of Euler-Lagrange partial differential equations. The first equation reads

$$
(1-W(x, y, z))(R-F(u, v, w)) \frac{\partial F}{\partial x}+\lambda \operatorname{div}(D(\nabla F) u)-\mu W(x, y, z)\left(u-u_{f}\right)=0
$$

with the other two showing the same basic structure. A semi-implicit scheme for solving the Euler-Lagrange equation makes use of a first-order Taylor approximation of $F(x+u, y+v, z+w)$. After discretization using standard finite difference stencils a huge but sparse system of linear equations has to be solved. For this purpose the iterative Gauss-Seidel algorithm is utilized. The whole registration algorithm is performed in a multi-resolution manner to speed-up computation and to avoid local minima.

\section{$5 \quad$ Experiments and Results}

To assess the validity of the hybrid registration approach qualitative and quantitative evaluations were performed on synthetically transformed and clinical thorax CT data sets showing breathing motion. All experiments were done on a standard Windows notebook computer with $2 \mathrm{GHz}$ and $2 \mathrm{~GB}$ RAM.

\subsection{Synthetic Deformations}

Synthetic experiments were performed on four test data sets (A,B,C,D) taken at inspiration, each of them having a volume size of $256 \times 256 \times 256$ voxels. The 


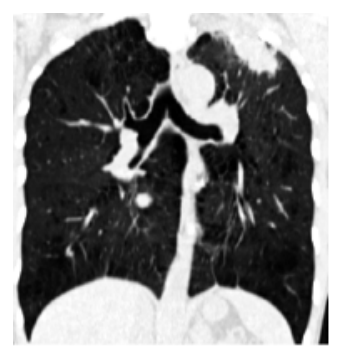

a)

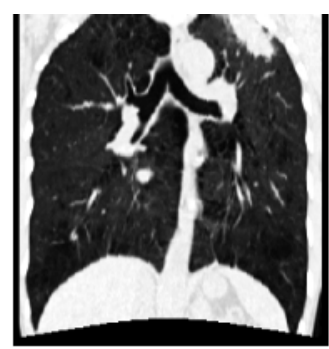

b)

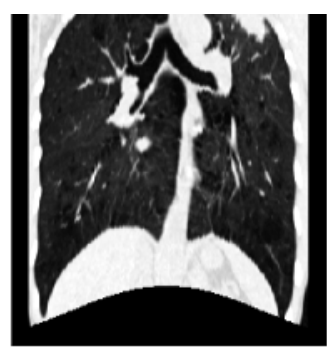

c)

Fig. 1. Simulated breathing deformation. a) original data set A, b) the small and c) the large deformation.

applied transformation intends to model a simple displacement field similar to exhalation. A synthetic nonlinear transformation $\boldsymbol{d}(x, y, z): \mathcal{R}^{3} \rightarrow \mathcal{R}^{3}$ that simulates diaphragm and rib cage movement has been presented by the authors in 5], due to space limitations the detailed derivation is omitted here. $\boldsymbol{d}$ is defined using two parameters $t_{\text {vertical }}$ and $t_{\text {inplane. }}$ The four test data sets were synthetically transformed with a small $\left(t_{\text {vertical }}=25 \mathrm{~mm}, t_{\text {inplane }}=10 \mathrm{~mm}\right)$ and a large $\left(t_{\text {vertical }}=55 \mathrm{~mm}, t_{\text {inplane }}=25 \mathrm{~mm}\right)$ deformation. Fig. 1 a)-c) shows the effects of these deformations on data set A. Table 1 gives the results of these experiments by comparing the hybrid approach (" hyb") with the landmarkbased approach ("lm") from Section 2 and the optical flow intensity registration ("of") from Section 3. All comparisons are always performed only on those regions which are present in both registered data sets. The RMS of the intensity differences $I N T_{r m s}$ before (" init") and after registration are calculated, as well as the difference of the resulting and the synthetic displacement fields in terms of the RMS of the whole displacement vector field $D F_{r m s}$ and the maximum of

Table 1. Simulated breathing transformation. Registration results in terms of the RMS of the intensity differences $I N T_{r m s}$, the RMS of the displacement field $D F_{r m s}$ and the maximum of the displacement difference vector components $D F_{\max }$.

\begin{tabular}{|c|c|c|c|c|c|c|c|c|c|}
\hline \multirow{2}{*}{\multicolumn{2}{|c|}{ Measure }} & \multicolumn{4}{|c|}{ Simulated Breathing 25-10 } & \multicolumn{4}{|c|}{ Simulated Breathing 55-25 } \\
\hline & & $\mathrm{A}$ & $\mathrm{B}$ & $\mathrm{C}$ & $\mathrm{D}$ & $\mathrm{A}$ & $\mathrm{B}$ & $\mathrm{C}$ & $\mathrm{D}$ \\
\hline init & [HU] & 373.85 & 313.70 & 291.08 & 348.11 & 539.98 & 468.57 & 438.07 & 513.42 \\
\hline है & [HU] & 77.045 & 75.084 & 4.972 & 75.529 & 123.43 & 06.01 & 102.27 & 116.022 \\
\hline -itm & [HU] & 61.888 & 61.891 & 55.351 & 69.595 & 149.69 & 137.94 & 105.22 & 178.82 \\
\hline$\checkmark h y b$ & {$[\mathrm{HU}]$} & 57.719 & 45.187 & 8.769 & 44.441 & 111.47 & 103.32 & 101.64 & 108.74 \\
\hline ही $o f$ & {$[\mathrm{~mm}]$} & 4.4201 & 6.0393 & 4.1328 & 4.6852 & 9.4332 & 12.428 & 8.3835 & 10.039 \\
\hline $\mathrm{lm}$ & {$[\mathrm{mm}]$} & 1.2700 & 1.6068 & 1.2759 & 1.3829 & 4.3041 & 4.7178 & 3.9078 & 4.6794 \\
\hline A $h y b$ & {$[\mathrm{~mm}]$} & 1.6826 & 2.4241 & 1.7129 & 2.1525 & 4.7948 & 5.7478 & 5.0956 & 5.7385 \\
\hline : & {$[\mathrm{mm}]$} & 19.926 & 22.976 & 16.925 & 18.366 & 40.826 & 48.667 & 40.868 & 43.429 \\
\hline ही $l m$ & {$[\mathrm{mn}$} & 22.712 & 21.981 & 21.586 & 22.219 & 49.008 & 50.426 & 47.969 & 49.375 \\
\hline - $h y b$ & {$[\mathrm{~mm}]$} & 17.488 & 19.770 & 16.489 & 17.909 & 35.090 & 36.737 & 32.985 & 41.322 \\
\hline
\end{tabular}


the displacement difference vector components $D F_{\max }$. Qualitative results are shown in terms of difference images of data set D in Fig. 3] a)-c).

\subsection{Clinical Data}

The algorithm was also evaluated on four clinical thoracic data sets (A,B,C,D), each of them consisting of two scans at different breathing states. The data sets show different problem characteristics. Data sets A and D differ by small breathing deformations. Data sets B and $\mathrm{C}$ differ by large breathing deformations and also show intensity variations due to diseases making them very hard to register. For the clinical data no gold standard displacement was available for comparison, therefore solely the decrease in the RMS of the intensity differences before and after registration are calculated and presented in Fig. 2, For qualitative results difference images are shown in Fig. 3 d)-e) for data sets C and D.

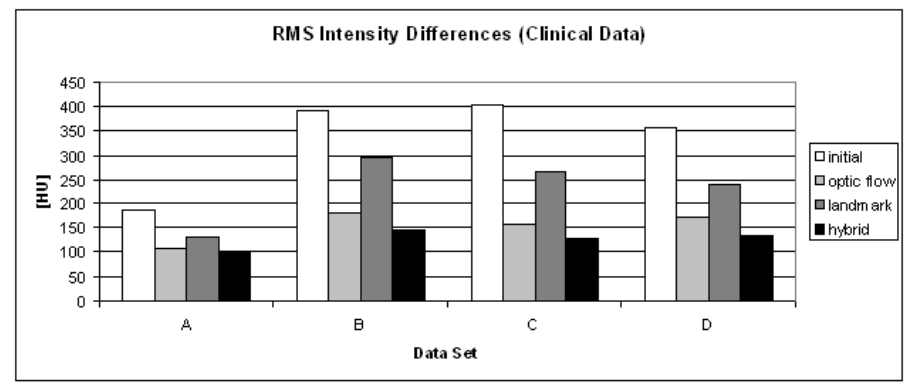

Fig. 2. Result chart showing the RMS of the intensity differences on the clinical data

\section{Discussion and Outlook}

The quantitative evaluation on the simulated data shows that the presented hybrid approach is an effective way to combine the advantages of landmark- and intensity-based registration. The RMS of the intensity differences on the small deformation data is slightly better than the individual methods, however all three methods are able to register these deformations very well. The displacement field outliers $\left(D F_{\max }\right)$ are also improved while the RMS of the displacement field $\left(D F_{r m s}\right)$ is slightly worse due to the influence of the intensity registration. However with an absolute value of $D F_{r m s}$ lying around $2 \mathrm{~mm}$ this is already a very accurate result. The large deformation data is more demanding to register. Here the landmark-based approach has problems generating a dense set of correspondences. On the other hand, due to the large imposed deformation, the intensity-based registration has problems to align small vascular structures. With the hybrid approach the best of both approaches is achieved which can be seen in the decrease of $I N T_{r m s}$ and on the qualitative results in Fig. 3 a)-c). The RMS of the displacement fields again decrease to a similar level as in the landmark registration and the displacement field outliers are reduced. The large absolute 

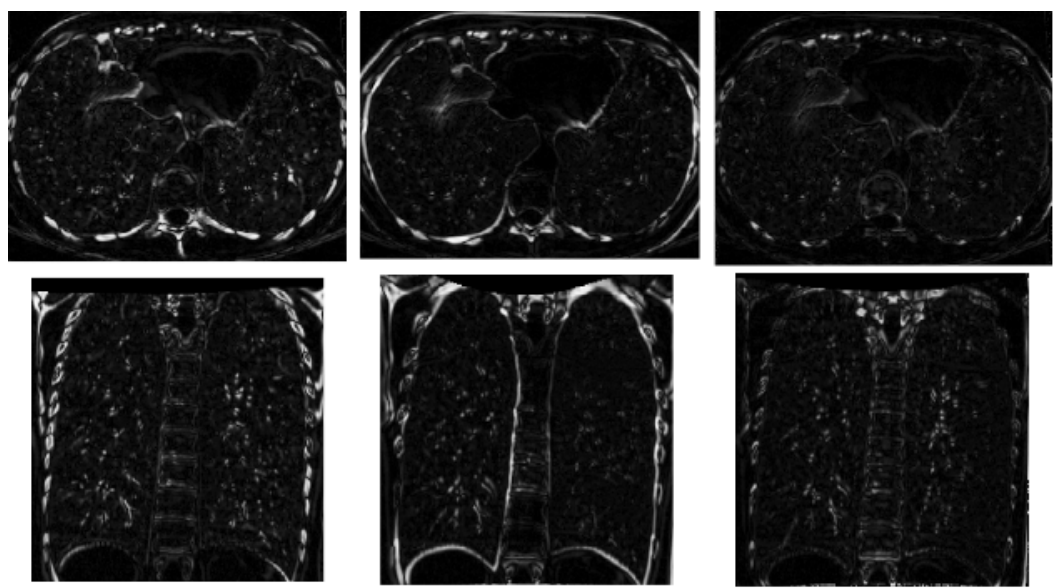

a)

b)

c)
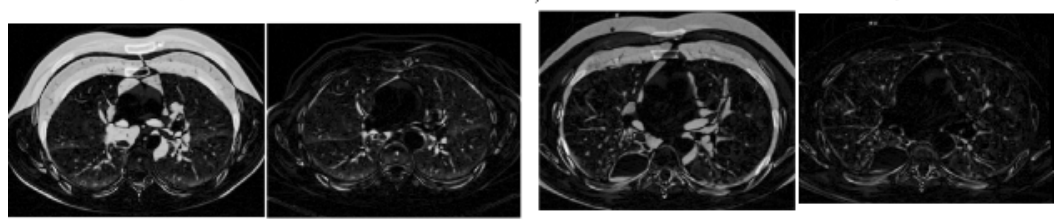

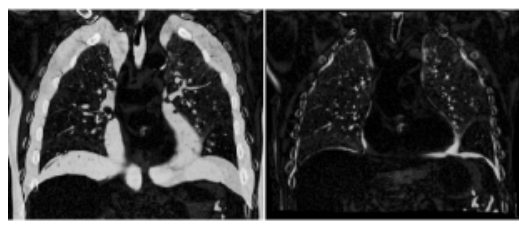

d)

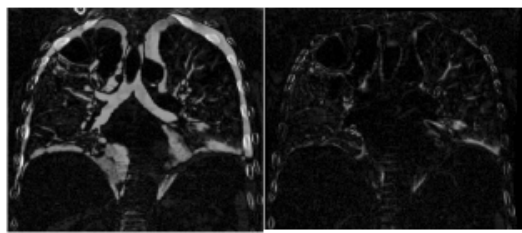

e)

Fig. 3. Selected Results. a)-c) Simulated Breathing results for the large deformation on data set D. a) shows difference images after optical flow, b) after landmark-based and c) after hybrid registration. d) depicts difference images of clinical data set $\mathrm{C}$ before and after hybrid registration, e) shows clinical data set D before and after hybrid registration. (Image contrast was enhanced to improve visibility.)

values of the displacement field outliers are due to problems at the border of the data sets. The clinical data also shows good correspondence after registration. However, registration of the vascular structures still can be improved. An important thing to consider is the proper choice of the algorithm parameters in the hybrid approach. Careful choice of parameters $\lambda$ and $\mu$ are crucial. In our experiments, after appropriate pre-normalization of data and regularization terms, suitable values were determined empirically in a set of initial experiments and remained fixed during the evaluations. In our case we chose $\lambda=0.05$ and $\mu=2$. For solving the Euler-Lagrange equations the linearizing Taylor approximation leads to the choice of a number of outer iterations. A trade-off of computation time and accuracy leads to more iterations on coarser resolutions compared to the finer ones. For the Gauss-Seidel stage three internal iterations were chosen. 
Concerning algorithm runtime, as expected, the landmark-based approach performed fastest (around 1000s), while the intensity- and the hybrid approaches take around 1900s. The overhead of incorporating the landmark correspondences only slightly increased the runtime. Intended future work will investigate methods to speed-up the time consuming stages, e.g. by a multi-grid approach. Further, different data and displacement regularization terms will be investigated in combination with the landmark-matching constraint.

\section{References}

1. Maintz, J., Viergever, M.: A Survey of Medical Image Registration. Medical Image Analysis 2(1) (1998) 1-36

2. Crum, W.R., Hartkens, T., Hill, D.L.G.: Non-rigid image registration: theory and practice. The British Journal of Radiology 77 (2004) 140-153

3. Rohr, K.: Landmark-Based Image Analysis Using Geometric and Intensity Models. Computational Imaging and Vision. Kluwer Academic Publishers (2001)

4. Bookstein, F.: Principal Warps: Thin-Plate Splines and the Decomposition of Deformations. IEEE PAMI 11(6) (1989) 567-585

5. Urschler, M., Bauer, J., Ditt, H., Bischof, H.: SIFT and Shape Context for FeatureBased Nonlinear Registration of Thoracic CT Images. In: Proc. Computer Vision Applications to Medical Image Analysis. (2006), to appear.

6. Gee, J.C.: Probabilistic Matching of Deformed Images. PhD thesis, Univ. Pennsylvania, Philadelphia (1996)

7. Johnson, H.J., Christensen, G.E.: Consistent Landmark and Intensity-Based Image Registration. IEEE TMI 21(5) (2002) 450-461

8. Li, B., Christensen, G.E., Fill, J., Hoffman, E.A., Reinhardt, J.M.: 3D Inter-Subject Warping and Registration of Pulmonary CT Images for a Human Lung Model. In: Proc SPIE Conf on Medical Imaging. Volume 4683. (2002) 324-335

9. Fan, L., Chen, C.W.: 3D Warping and Registration from Lung Images. In: Proc SPIE Conf on Medical Imaging. Volume 3660. (1999) 459-470

10. Hellier, P., Barillot, C.: Coupling Dense and Landmark Based Approaches for Nonrigid Registration. IEEE TMI 22(2) (2003) 217-227

11. Liu, T., Shen, D., Davatzikos, C.: Deformable registration of cortical structures via hybrid volumetric and surface warping. NeuroImage 22 (2004) 1790-1801

12. Papademetris, X., Jackowski, A.P., Schultz, R.T., Staib, L.H., S., D.J.: Integrated Intensity and Point-Feature Nonrigid Registration. In: Proc MICCAI. Volume LNCS 3216, Springer (2004) 763-770

13. Modersitzki, J.: Numerical Methods for Image Registration. Oxford University Press (2004)

14. Mikolajczyk, K., Schmid, C.: Performance Evaluation of Local Descriptors. IEEE PAMI 27(10) (2005) 1615-1630

15. Lowe, D.G.: Distinctive Image Features from Scale-Invariant Keypoints. Int J Computer Vision 60(2) (2004) 91-110

16. Belongie, S., Malik, J., Puzicha, J.: Shape matching and object recognition using shape contexts. IEEE PAMI 24(4) (2002) 509-522

17. Weickert, J., Schnörr, C.: A Theoretical Framework for Convex Regularizers in PDE-Based Computation of Image Motion. Int J Computer Vision 45(3) (2001) $245-264$ 